PROCEEDINGS OF THE

AMERICAN MATHEMATICAL SOCIETY

Volume 131, Number 4, Pages 1225-1231

S 0002-9939(02)06663-7

Article electronically published on July 26, 2002

\title{
A REMARK ON QUASI-ISOMETRIES
}

\author{
N. J. KALTON
}

(Communicated by N. Tomczak-Jaegermann)

\begin{abstract}
We show that if $f: B_{n} \rightarrow \mathbb{R}^{n}$ is an $\epsilon$-quasi-isometry, with $\epsilon<1$, defined on the unit ball $B_{n}$ of $\mathbb{R}^{n}$, then there is an affine isometry $h: B_{n} \rightarrow \mathbb{R}^{n}$ with $\|f(x)-h(x)\| \leq C \epsilon(1+\log n)$ where $C$ is a universal constant. This result is sharp.
\end{abstract}

\section{InTROdUCTION}

Suppose $\epsilon>0$ and $X$ and $Y$ are Banach spaces and let $B_{X}=\{x:\|x\| \leq 1\}$. An $\epsilon$-quasi-isometry $f: B_{X} \rightarrow Y$ is a local homeomorphism such that for any $x \in B_{X}$ we have

$$
(1+\epsilon)^{-1} \leq \liminf _{y \rightarrow x} \frac{\|f(y)-f(x)\|}{\|y-x\|} \leq \limsup _{y \rightarrow x} \frac{\|f(y)-f(x)\|}{\|y-x\|} \leq(1+\epsilon) .
$$

A map $f: B_{X} \rightarrow Y$ is $\epsilon$-rigid if

$$
(1+\epsilon)^{-1}\left\|x_{1}-x_{2}\right\| \leq\left\|f\left(x_{1}\right)-f\left(x_{2}\right)\right\| \leq(1+\epsilon)\left\|x_{1}-x_{2}\right\|, \quad x_{1}, x_{2} \in B_{X} .
$$

If $\operatorname{dim} X=\operatorname{dim} Y<\infty$, then any $\epsilon$-rigid map is also an $\epsilon$-quasi-isometry (cf. [8]). We refer to [2] for a full discussion.

In this note we will be concerned with the case when $X=Y=\mathbb{R}^{n}$ with the usual Euclidean norm (we then write $B_{n}$ for the closed unit ball in $\mathbb{R}^{n}$ ). In this context John [6] (discussed in 2]) showed that if $f$ is an $\epsilon$-quasi-isometry one can find an $a \in \mathbb{R}^{n}$ and an orthogonal linear operator $U$ so that

$$
\|f(x)-U x-a\| \leq C n^{\frac{3}{2}} \epsilon, \quad x \in B_{n} .
$$

See also a recent paper [1] for related results. Recently the exponent $\frac{3}{2}$ has been improved to $\frac{1}{2}$ by Vestfrid [11]. This raises the question of finding the optimal function $\alpha(n)$ so that given any $\epsilon$-quasi-isometry $f$ one can find an orthogonal $U$ and $a$ such that

$$
\|f(x)-U x-a\| \leq \alpha(n) \epsilon, \quad x \in B_{n} .
$$

In another direction Matouskova [8] has given examples to show that $\alpha(n) \geq c \log n$ for any such function. The aim of this note is to show that this lower estimate is in fact sharp and we can take $\alpha(n) \leq C(\log n+1)$.

Received by the editors June 10, 2001 and, in revised form, November 27, 2001.

2000 Mathematics Subject Classification. Primary 46C05, 47H99.

Key words and phrases. Quasi-isometries in Euclidean spaces.

The author was supported by NSF grant DMS-9870027. 
In the final section we make a few remarks on the connections between this result and the theory of twisted sums, and make an observation about an old problem of Figiel, Lindenstrauss and Milman.

\section{MAIN RESULTS}

Let us first discuss the example which gives the lower bound (due to Matouskova [8]). Fix $\epsilon>0$. Assume $n$ is even, say $n=2 m$, and identify $\mathbb{R}^{n}$ with $\mathbb{C}^{m}$. For $z=r e^{i \theta} \in \mathbb{C}$ let $\varphi_{\epsilon}(z)=z r^{i \epsilon}$ (if $z=0$, then $\varphi_{\epsilon}(z)=0$ ). Then $\varphi_{\epsilon}$ is an $\epsilon$-quasiisometry of $\mathbb{C}$ (see [6], [2] p. 352) and so it follows easily that if we set

$$
f_{\epsilon}\left(z_{1}, \ldots, z_{m}\right)=\left(\varphi_{\epsilon}\left(z_{1}\right), \ldots, \varphi_{\epsilon}\left(z_{m}\right)\right)
$$

then $f_{\epsilon}$ is also an $\epsilon$-quasi-isometry of $B_{n}$ onto $B_{n}$.

Proposition 2.1. Suppose $\epsilon \log m<2 \pi$. Then for any affine isometry $h(x)=$ $U x+a$ we have

$$
\max _{x \in B_{n}}\left\|f_{\epsilon}(x)-h(x)\right\| \geq \sin \left(\frac{1}{4} \epsilon \log m\right) .
$$

Remark 1. Let us remark that this example of Matouskova shows that Theorem 3 of [10] is incorrect, as was pointed out to us by Igor Vestrid.

Proof. Since $f_{\epsilon}(x)=-f_{\epsilon}(-x)$ we can clearly replace $h$ by $\frac{1}{2}(h(x)-h(-x))$ and so assume $h(x)=U x$. Note that $U$ is only assumed real-linear on $\mathbb{C}^{m}$. Let us suppose $U$ is the best linear approximation to $f_{\epsilon}$ (not necessarily orthogonal). Let $G$ be the group of unitary operators $V$ on $\mathbb{C}^{m}$ of the form $V\left(z_{1}, \ldots, z_{m}\right)=\left(u_{1} z_{\pi(1)}, \ldots, u_{m} z_{\pi(m)}\right)$ where $\left|u_{j}\right|=1$ for $1 \leq j \leq m$ and $\pi$ is a permutation of $\{1,2, \ldots, m\}$. Note that $V^{-1} f_{\epsilon}\left(V\left(z_{1}, \ldots, z_{m}\right)\right)=f_{\epsilon}\left(z_{1}, \ldots, z_{m}\right)$. Hence if $U$ is an optimal approximation we can replace it by

$$
U^{\prime}=\int_{G} V^{-1} U V d \mu(V)
$$

where $\mu$ is the Haar measure of $G$, and then $U^{\prime}$ is also optimal. Now $U^{\prime}$ commutes with each $V \in G$ and so is complex linear and furthermore must be of the form $r e^{i \theta} I$ where $I$ is the identity. Now

$$
\left\|f_{\epsilon}(1,0, \ldots, 0)-\left(r e^{i \theta}, 0, \ldots, 0\right)\right\| \geq|\sin \theta|
$$

and

$$
\left\|f_{\epsilon}\left(m^{-\frac{1}{2}}, m^{-\frac{1}{2}}, \ldots, m^{-\frac{1}{2}}\right)-r e^{i \theta}\left(m^{-\frac{1}{2}}, \ldots, m^{-\frac{1}{2}}\right)\right\| \geq\left|\sin \left(\frac{1}{2} \epsilon \log m-\theta\right)\right| .
$$

Clearly this leads to the result.

The following result is essentially contained in [3], but we will give some details here for completeness and ease of the reader. As we point out in the final section this result is in some sense derivable from classical results on Banach space theory and the theory of twisted sums, although to do it this way would arguably use more sophisticated notions than we really need.

Theorem 2.2. There is an absolute constant $C$ with the following property. Let $\Omega: \mathbb{R}^{n} \rightarrow \mathbb{R}^{n}$ be a continuous map which satisfies the conditions:

(1) $\Omega(\lambda x)=\lambda x, \quad \lambda \in \mathbb{R}, x \in \mathbb{R}^{n}$,

(2) $\left\|\Omega\left(x_{1}+x_{2}\right)-\Omega\left(x_{1}\right)-\Omega\left(x_{2}\right)\right\| \leq\left\|x_{1}\right\|+\left\|x_{2}\right\|, \quad x_{1}, x_{2} \in \mathbb{R}^{n}$.

Then there is a linear map $A: \mathbb{R}^{n} \rightarrow \mathbb{R}^{n}$ with $\|\Omega(x)-A(x)\| \leq C(\log n+1)\|x\|$ for all $x \in \mathbb{R}^{n}$. 
Proof. This theorem is almost proved in [3], but we will sketch the details. We need the following result, which is a fairly simple deduction from the Hahn-Banach Theorem (see Theorem 3.1 of [3] ):

Proposition 2.3. Suppose $K$ is a compact convex subset of $\mathbb{R}^{m}$ and that $f: K \rightarrow$ $\mathbb{R}$ is a continuous map satisfying the condition

$$
\left|f\left(\frac{1}{2}\left(x_{1}+x_{2}\right)\right)-\frac{1}{2}\left(f\left(x_{1}\right)+f\left(x_{2}\right)\right)\right| \leq 1, \quad x_{1}, x_{2} \in K .
$$

Then there is an affine map $a: K \rightarrow \mathbb{R}$ such that

$$
|f(x)-a(x)| \leq \log _{2} n+\frac{5}{2}, \quad x \in K .
$$

Returning to the proof of Theorem 2.2 we will first prove that there is a symmetric linear operator $B$ so that

$$
|\langle x, \Omega(x)-B x\rangle| \leq 5\left(\log _{2} n+1\right)\|x\|^{2} .
$$

We define $\varphi(x)=\langle x, \Omega(x)\rangle$. Let us observe that $\varphi(\lambda x)=\lambda^{2} \varphi(x)$ for $\lambda \in \mathbb{R}$ and $x \in \mathbb{R}^{n}$. Also, for $x_{1}, x_{2} \in \mathbb{R}^{n}$,

$$
\begin{aligned}
\varphi\left(x_{1}+x_{2}\right)+\varphi\left(x_{1}-x_{2}\right)=\langle & \left.x_{1}, \Omega\left(x_{1}+x_{2}\right)+\Omega\left(x_{1}-x_{2}\right)\right\rangle \\
& +\left\langle x_{2}, \Omega\left(x_{1}+x_{2}\right)-\Omega\left(x_{1}-x_{2}\right)\right\rangle
\end{aligned}
$$

and hence

$$
\begin{aligned}
\left|\frac{1}{2}\left(\varphi\left(x_{1}+x_{2}\right)+\varphi\left(x_{1}-x_{2}\right)\right)-\varphi\left(x_{1}\right)-\varphi\left(x_{2}\right)\right| & \leq\left(\left\|x_{1}\right\|+\left\|x_{2}\right\|\right)^{2} \\
& \leq 2\left(\left\|x_{1}\right\|^{2}+\left\|x_{2}\right\|^{2}\right) .
\end{aligned}
$$

Then if $P$ is a positive-definite operator we define $\nu_{P}$ to be the probability measure on $\mathbb{R}^{n}$ with Fourier transform

$$
\int_{\mathbb{R}^{n}} e^{-i\langle x, \xi\rangle} d \nu_{P}(x)=e^{-\frac{1}{2}\langle P \xi, \xi\rangle} .
$$

For each positive definite matrix $P$ we define

$$
f(P)=\int_{\mathbb{R}^{n}} \varphi(x) d \nu_{P}(x) .
$$

Observe that $f(\lambda P)=\lambda f(P)$ if $\lambda \geq 0$. Since $\nu_{P+Q}=\nu_{P} * \nu_{Q}$

$$
f(P+Q)=\int_{\mathbb{R}^{n}} \int_{\mathbb{R}^{n}} \varphi\left(x_{1}+x_{2}\right) d \nu_{P}\left(x_{1}\right) d \nu_{Q}\left(x_{2}\right) .
$$

By symmetry

$$
\begin{aligned}
f(P+Q) & =\int_{\mathbb{R}^{n}} \int_{\mathbb{R}^{n}} \varphi\left(x_{1}+x_{2}\right) d \nu_{P}\left(x_{1}\right) d \nu_{Q}\left(x_{2}\right) \\
& =\int_{\mathbb{R}^{n}} \int_{\mathbb{R}^{n}} \frac{1}{2}\left(\varphi\left(x_{1}+x_{2}\right)+\varphi\left(x_{1}-x_{2}\right)\right) d \nu_{P}\left(x_{1}\right) d \nu_{Q}\left(x_{2}\right) \\
& =\rho+\int_{\mathbb{R}^{n}} \int_{\mathbb{R}^{n}}\left(\varphi\left(x_{1}\right)+\varphi\left(x_{2}\right)\right) d \nu_{P}\left(x_{1}\right) d \nu_{Q}\left(x_{2}\right)
\end{aligned}
$$

where

$$
|\rho| \leq 2 \int_{\mathbb{R}^{n}} \int_{\mathbb{R}^{n}}\left(\left\|x_{1}\right\|^{2}+\left\|x_{2}\right\|^{2}\right) d \nu_{P}\left(x_{1}\right) d \nu_{Q}\left(x_{2}\right) \leq 2 \operatorname{tr}(P+Q) .
$$

Now let $\Gamma_{0}=\{P: \operatorname{tr} P=1\}$. Then for $P, Q \in \Gamma_{0}$ we have

$$
\left|f\left(\frac{1}{2}(P+Q)\right)-\frac{1}{2}(f(P)+f(Q))\right| \leq 2 .
$$


Now $\Gamma_{0}$ is a compact convex subset in the $\frac{1}{2} n(n+1)$-dimensional space $\Sigma_{n}$ of symmetric matrices and so by Proposition $\left[2.3\right.$ we have an affine map $a: \Gamma_{0} \rightarrow \mathbb{R}$ such that

$$
|f(P)-a(P)| \leq 5\left(\log _{2} n+1\right), \quad P \in \Gamma_{0} .
$$

Extending $a$ by homogeneity we obtain a linear map $h$ on the space $\Sigma_{n}$ so that

$$
|f(P)-h(P)| \leq 5\left(\log _{2} n+1\right) \operatorname{tr} P
$$

if $P \geq 0$. For $x \in \mathbb{R}^{n}$ let $x \otimes x$ be the rank-one operator $y \rightarrow\langle y, x\rangle x$. Then $q(x)=h(x \otimes x)$ is a quadratic form and so there is a symmetric operator $S$ so that $q(x)=\langle x, S x\rangle$ for $x \in \mathbb{R}^{n}$. Now $f(x \otimes x)=\varphi(x)$ and so we conclude that (2.1) holds.

To complete the proof we consider $\mathbb{R}^{2 n}=\mathbb{R}^{n} \times \mathbb{R}^{n}$ and define $\Omega^{\prime}(\mathbf{x})=\left(0, \Omega\left(x_{1}\right)\right)$ if $\mathbf{x}=\left(x_{1}, x_{2}\right)$. By (2.1) we find a symmetric $2 n \times 2 n$ matrix $B$ so that

$$
\left|\left\langle\mathbf{x}, \Omega^{\prime}(\mathbf{x})-B(\mathbf{x})\right\rangle\right| \leq 5\left(\log _{2} n+2\right)\|\mathbf{x}\|^{2} .
$$

Let

$$
B(\mathbf{x})=\left(B_{11} x_{1}+B_{12} x_{2}, B_{21} x_{1}+B_{22} x_{2}\right)
$$

where $B_{j k}$ are $n \times n$ matrices for $1 \leq j, k \leq 2$. Then if $\epsilon_{1}, \epsilon_{2}= \pm 1$ we obtain

$$
\left|\left\langle x_{2}, \Omega\left(x_{1}\right)\right\rangle-\epsilon_{1} \epsilon_{2} \sum_{j, k \leq 2} \epsilon_{j} \epsilon_{k}\left\langle x_{j}, B_{j k} x_{k}\right\rangle\right| \leq 5\left(\log _{2} n+2\right)\left(\left\|x_{1}\right\|^{2}+\left\|x_{2}\right\|^{2}\right) .
$$

Averaging over choices of sign and noting that $B_{12}=B_{21}^{t}$ we obtain (if $A=2 B_{21}$ )

$$
\left|\left\langle x_{2}, \Omega\left(x_{1}\right)-A x_{1}\right\rangle\right| \leq 5\left(\log _{2} n+2\right)\left(\left\|x_{1}\right\|^{2}+\left\|x_{2}\right\|^{2}\right), \quad x_{1}, x_{2} \in \mathbb{R}^{n} .
$$

Taking $\left\|x_{1}\right\|=\left\|x_{2}\right\|=1$ and using homogeneity, this leads to the inequality

$$
\|\Omega(x)-A x\| \leq 10\left(\log _{2} n+2\right)\|x\|, \quad x \in \mathbb{R}^{n} .
$$

This completes the proof.

The following lemma follows from Lemma 2.5 of Matouskova 8]; a more general lemma for arbitrary Banach spaces has been proved by Vestfrid [11].

Lemma 2.4. Suppose $f: B_{n} \rightarrow \mathbb{R}^{n}$ is an $\epsilon$-quasi-isometry where $\epsilon<10^{-2}$. Then if $\left\|x_{1}\right\|,\left\|x_{2}\right\| \leq \frac{1}{2}$

$$
\left\|f\left(\frac{1}{2}\left(x_{1}+x_{2}\right)\right)-\frac{1}{2}\left(f\left(x_{1}\right)+f\left(x_{2}\right)\right)\right\| \leq 65 \epsilon\left\|x_{1}-x_{2}\right\| .
$$

Lemma 2.5. Let $g:[0,1] \rightarrow \mathbb{R}$ be a continuous function which satisfies

$$
\left|g(t)-\frac{1}{2}(g(t+s)+g(t-s))\right| \leq s, \quad 0<s \leq \min \left(t, \frac{1}{2}(1-t)\right) .
$$

Then

$$
\max _{0 \leq t \leq 1}|g(t)| \leq 14+12 \max _{0 \leq t \leq \frac{1}{2}}|g(t)| .
$$

Proof. Let $M=\max _{0 \leq x \leq \frac{1}{2}}|g(x)|$. It will suffice to show that $g(t) \leq 12 M+14$ since then one can obtain the same estimate for $-g$.

Let $h(t)=(1-t) \log (1-t)($ with $h(1)=0)$. Note $h$ is continuous and $-\frac{1}{2} \leq$ $h(t) \leq 0$ for $0 \leq t \leq 1$. We also observe that

$$
\frac{3}{2} t \log \frac{3}{2} t+\frac{1}{2} t \log \frac{1}{2} t=2 t \log t+t \log \frac{3 \sqrt{3}}{4} .
$$


Then if $\frac{1}{3} \leq t \leq 1$ we have

$$
\frac{1}{2}\left(h\left(\frac{1}{2}(3 t-1)\right)+h\left(\frac{1}{2}(1+t)\right)\right) \geq h(t)+\frac{1}{8}(1-t)
$$

since $\log \frac{3 \sqrt{3}}{4}>\frac{1}{4}$. Note that if $g$ satisfies (2.2),

$$
\frac{1}{2}\left(g\left(\frac{1}{2}(3 t-1)\right)+g\left(\frac{1}{2}(1+t)\right)\right) \geq g(t)-\frac{1}{2}(1-t) .
$$

It follows from (2.3) and (2.4) that if $g$ satisfies (2.2), then $g+4 h$ cannot have a maximum for $\frac{1}{3} \leq t<1$. This implies that

$$
g(t) \leq \max \left(\max _{0 \leq x \leq \frac{1}{3}} g(x), g(1)\right)+2, \quad 0 \leq t \leq 1 .
$$

If $g(1) \leq 0$, then we immediately obtain $g(t) \leq M+2$ for $0 \leq t \leq 1$ and the proof is complete. If $g(1)>0$, we define $f(t)=\operatorname{tg}(1)-g(t)$ and note that $f$ also satisfies (2.2). For $0 \leq t \leq \frac{1}{3}$ we have $f(t) \leq M+\frac{1}{3} g(1)$ and we also have $f(1)=0$. By 2.5

$$
f(t) \leq \frac{1}{3} g(1)+M+2 .
$$

Letting $t=\frac{1}{2}$ we have

$$
\frac{1}{6} g(1) \leq g\left(\frac{1}{2}\right)+M+2 \leq 2 M+2 .
$$

Hence $g(1) \leq 12 M+12$ and we now use (2.5) to complete the proof.

Theorem 2.6. There is a universal constant $C$ with the following property. Let $f: B_{n} \rightarrow \mathbb{R}^{n}$ be an $\epsilon$-quasi-isometry where $0<\epsilon<1$. Then there exists $a \in \mathbb{R}^{n}$ and an orthogonal linear map $U$ with

$$
\|f(x)-U x-a\| \leq C \epsilon(\log n+1)
$$

for $x \in B_{n}$.

Proof. It suffices to consider the case $\epsilon<10^{-2}$. We may assume $f(0)=0$. Let us then define

$$
\Omega(x)=\|x\|\left(f\left(\frac{x}{2\|x\|}\right)-f\left(-\frac{x}{2\|x\|}\right)\right), \quad x \in \mathbb{R}^{n} .
$$

$\Omega$ is clearly homogeneous. Next suppose $\|x\|=1$ and $\|y\|=1$. Define $h(t)=$ $\langle f(t x)-\Omega(t x), y\rangle$ for $-1 \leq t \leq 1$. Note that by Lemma 2.4 applied to the ball $t x+(1-|t|) B_{X}$, we have

$$
\left|h(t)-\frac{1}{2}(h(t+s)+h(t-s))\right| \leq 200 \epsilon s, \quad 0<s \leq \min \left(|t|, \frac{1}{2}(1-|t|)\right) .
$$

In particular if we apply the Whitney Lemma ([12]) there is an affine function $a$ so that $|h(t)-a(t)| \leq 100 \epsilon$ for $-\frac{1}{2} \leq t \leq \frac{1}{2}$. Now

$$
f\left( \pm \frac{1}{2} x\right)-\Omega\left( \pm \frac{1}{2} x\right)=\frac{1}{2}\left(f\left(\frac{1}{2} x\right)+f\left(-\frac{1}{2} x\right)\right) .
$$

Then $h\left(\frac{1}{2}\right)=h\left(-\frac{1}{2}\right)$ and by Lemma 2.4, $\left|h\left(\frac{1}{2}\right)\right| \leq 65 \epsilon$. Thus

$$
|a(t)| \leq\left|h\left(\frac{1}{2}\right)\right|+100 \epsilon \leq 200 \epsilon
$$

for $0 \leq t \leq \frac{1}{2}$. Hence

$$
|h(t)| \leq 300 \epsilon, \quad|t| \leq \frac{1}{2} .
$$

Now by Lemma 2.5 (used on both $[0,1]$ and $[-1,0]$ ) we obtain

$$
|h(t)| \leq C_{0} \epsilon, \quad|t| \leq 1
$$


where $C_{0}<10^{4}$. We conclude that

$$
\|f(x)-\Omega(x)\| \leq C_{0} \epsilon, \quad\|x\| \leq 1 .
$$

Now if $x_{1}, x_{2} \in \mathbb{R}^{n}$ we define $b=2\left(\left\|x_{1}\right\|+\left\|x_{2}\right\|\right)$ and then note that

$$
\begin{aligned}
& \left\|\Omega\left(x_{1}+x_{2}\right)-\Omega\left(x_{1}\right)-\Omega\left(x_{2}\right)\right\| \\
& =b\left\|\Omega\left(b^{-1}\left(x_{1}+x_{2}\right)\right)-\Omega\left(b^{-1} x_{1}\right)-\Omega\left(b^{-1} x_{2}\right)\right\| \\
& \leq C_{0} \epsilon b+b\left\|2 f\left(\frac{1}{2}\left(b^{-1}\left(x_{1}+x_{2}\right)\right)\right)-f\left(b^{-1} x_{1}\right)-f\left(b^{-1} x_{2}\right)\right\| \\
& \leq C_{0} \epsilon b+200 \epsilon\left\|x_{1}-x_{2}\right\| \leq C_{1} \epsilon\left(\left\|x_{1}\right\|+\left\|x_{2}\right\|\right)
\end{aligned}
$$

where $C_{1}=2 C_{0}+200$.

Now we can apply Theorem 2.2 to deduce the existence of a linear map $A$ so that

$$
\|\Omega(x)-A x\| \leq C_{2}(1+\log n) \epsilon\|x\|, \quad x \in \mathbb{R}^{n},
$$

for some absolute constant $C_{2}$. If $\|x\| \leq 1$ we have

$$
\|f(x)-A x\| \leq\left(C_{2}(1+\log n)+C_{0}\right) \epsilon
$$

and hence if $\|x\|=1$ we have

$$
1-C_{3}(\log n+1) \epsilon \leq\|A x\| \leq 1+C_{3}(\log n+1)
$$

where $C_{3}$ is a universal constant. This implies there is an orthogonal transformation $U$ with $\|A-U\| \leq C_{3}(\log n+1)$. Then $U$ satisfies the conclusion of the theorem.

\section{REMARKS}

In fact Theorem 2.2 can be reached from known results in Banach space theory using the theory of twisted sums (and more specifically twisted Hilbert spaces). We sketch the ideas. One can use the map $\Omega$ to define a $(2 n)$-dimensional Banach space $X$ with a Euclidean subspace $E$ with $\operatorname{dim} E=n$ so that $X / E$ is also Euclidean. It then follows from results of Figiel, Lindenstrauss and Milman [5] (see also [4]) that $X$ has type 2 constant $T_{2}(X) \leq C(1+\log n)$. But then Maurey's extension theorem [9] produces a projection $P: X \rightarrow E$ with $\|P\| \leq T_{2}(X)$. This projection induces the linear approximation $A$ in the standard way.

We would like to take this opportunity to resolve a question raised in [5] concerning such twisted sums. In [5], Figiel, Lindenstrauss and Milman ask for an estimate on the Banach-Mazur distance $d_{X}=d\left(X, \ell_{2}^{2 n}\right)$ when $X$ is as above (i.e. $\operatorname{dim} X=2 n$ and $X$ has a subspace $E$ of dimension $n$ with $E$ and $X / E$ isometrically Euclidean). They note that in [4] an example is given with $d_{X} \geq c(\log n)^{\frac{1}{2}}$ and they obtain an upper estimate of the type $C(\log n+1)^{2}$. Later in [7] an example was produced with $d_{X} \geq c \log n$. We now point out that this is sharp, as the upper estimate can be improved to $C(\log n+1)$. Curiously this requires nothing other than the results of [5]. The bound established in [5] uses Kwapien's theorem and a logarithmic estimate on both type and cotype; by using Maurey's theorem one eliminates a logarithmic factor.

Theorem 3.1. There is a universal constant $C$ such that if $X$ is a Banach space of dimension $2 n$ with a subspace $E$ of dimension $n$ with $E, X / E$ isometrically Euclidean, then $d\left(X, \ell_{2}^{2 n}\right) \leq C(1+\log n)$. 
Proof. As remarked above $T_{2}(X) \leq C(1+\log n)$ for some universal constant $C$ (see Theorem 6.5 of [5]). By Maurey's extension theorem [9] there is a projection $P: X \rightarrow E$ with $\|P\| \leq T_{2}(X)$. Let $Q$ be the quotient map $Q: X \rightarrow X / E$. Define a Euclidean norm on $X$ by

$$
\|x\|_{H}^{2}=\frac{1}{2}\left(\|P\|^{-2}\|P x\|^{2}+\|Q x\|^{2}\right) .
$$

Then $\|x\|_{E} \leq\|x\|$. Conversely assume $\|x\| \geq 8\|P\|\|x\|_{H}$. Then $\|Q x\| \leq \frac{1}{4}\|P\|^{-1}\|x\|$. Pick $e \in E$ so that $\|x-e\|=\|Q x\|$. Then

$$
\|P x\| \geq\|e\|-\|P(x-e)\| \geq\|x\|-\|x-e\|(1+\|P\|) .
$$

Hence

$$
\|P x\| \geq \frac{3\|P\|-1}{4\|P\|}\|x\| \geq \frac{1}{2}\|x\|
$$

and

$$
\|x\|_{H} \geq \frac{1}{2\|P\|}\|x\|
$$

which is a contradiction. Thus

$$
\|x\|_{H} \leq\|x\| \leq 8 T_{2}(X)\|x\|
$$

and the proof is complete.

\section{REFERENCES}

[1] P. Alestalo, D.A. Trotsenko and J. Väisaäla, Isometric approximation, Israel J. Math. 125 (2001) 61-82.

[2] Y. Benyamini and J. Lindenstrauss, Geometric nonlinear functional analysis, Volume 1 Colloquium Publications No. 48, Amer. Math. Soc. 2000. MR 2001b:46001

[3] Y. Brudnyi and N.J. Kalton, Polynomial approximation on convex subsets of $\mathbf{R}^{\mathbf{n}}$, Constructive Approximation 16 (2000) 161-200. MR 2000k:41036

[4] P. Enflo, J. Lindenstrauss and G. Pisier, On the three space problem, Math. Scand. 36 (1975) 199-210. MR 52:3928

[5] T. Figiel, J. Lindenstrauss and V.D. Milman, The dimension of almost spherical sections of convex bodies, Acta. Math. 139 (1977) 53-94. MR 56:3618

[6] F. John, Rotation and strain, Comm. Pure. Appl. Math. 14 (1961) 391-413. MR 25:1672

[7] N.J. Kalton and N.T. Peck, Twisted sums of sequence spaces and the three space problem, Trans. Amer. Math. Soc. 255 (1979) 1-30. MR 82g:46021

[8] E. Matouskova, Almost isometries of balls, J. Functional Analysis 190 (2002) 507-525.

[9] B. Maurey, Une théorème de prolongement, C.R. Acad. Sci Paris 279 (1974) 329-332. MR 50:8013

[10] V.I. Semenov, Estimates of stability for spatial quasiconfromal mappings of a starlike region, Siberian Math. J. 28 (1987) 946-955.

[11] I.A. Vestfrid, Affine properties and injectivity of quasi-isometries, to appear.

[12] H. Whitney, On functions with bounded $n$th differences, J. Math. Pures Appl. 36 (1957) 67-95. MR 18:889f 65211

Department of Mathematics, University of Missouri-Columbia, Columbia, Missouri

E-mail address: nigel@math.missouri.edu 